\title{
Are the distributions of carbohydrates, meals and snacks associated with blood glucose control in women with gestational diabetes? A pilot study using myfood24 dietary assessment tool
}

\author{
J. Hutchinson ${ }^{1}$, M. Morris ${ }^{1}$, C. Gianfrancesco ${ }^{1}$, N.A. Alwan ${ }^{2}$ and J.E. Cade ${ }^{1}$ \\ ${ }^{1}$ Nutritional Epidemiology Group, School of Food Science and Nutrition, University of Leeds and \\ ${ }^{2}$ Academic Unit of Primary Care and Population Sciences, University of Southampton
}

Women with gestational diabetes mellitus (GDM) have a higher risk of maternal and fetal complications than healthy pregnant women $^{(1)}$. To reduce this risk, the latest guidance is to achieve fasting blood glucose (FBG) levels below $5 \cdot 3 \mathrm{mmol} / 1$ and 1 hour post meal levels of below $7.8 \mathrm{mmol} / \mathrm{l}^{(2)}$. First line treatment is to encourage a healthy diet including carbohydrate (CHO) management; but evidence is lacking for a recommended daily $\mathrm{CHO}$ distribution ${ }^{(3)}$. Our aim was to explore $\mathrm{CHO}$, meal and snack distribution in relation to blood glucose control.

200 women recently diagnosed with GDM (aged 20-43 years) attending the Diabetes Antenatal Clinic at Leeds Teaching Hospitals NHS Trust recorded their diet for up to five days using myfood $24-$ a new online 24 h dietary recall tool ${ }^{(4)}$. 115 women also recorded their fasting blood glucose (FBG) for up to seven days (and 103 recorded post-meal blood glucose). All continued to receive usual pregnancy care for women with GDM. For the analyses, women were split into two groups: (A) those achieving the FBG target $(<5.3 \mathrm{mmol} / \mathrm{L})$ and $(\mathrm{B})$ those who did not on average over the blood sampling days. Analysis determined whether there were significant differences between groups (A) and (B) in relation to i) frequency of meal consumption (using Chi ${ }^{2}$ tests), ii) distribution of calories and $\mathrm{CHO}$ over six eating periods across the day (using Mann-Whitney tests), iii) percentage of meals and snacks with 30 to $50 \mathrm{~g} \mathrm{CHO} / \mathrm{meal}$ and $\leqslant 20 \mathrm{~g}$ of $\mathrm{CHO} / \mathrm{snack}$ period (often advised in practise). Similar analyses were undertaken to compare women who achieved post-breakfast blood glucose target with those who did not.

\begin{tabular}{|c|c|c|c|c|c|}
\hline \multirow[b]{2}{*}{ Distribution of carbohydrate intake $\%$} & \multicolumn{2}{|c|}{$\begin{array}{l}\text { Achieved FBG } \\
\text { target (A) } \mathrm{N}=88\end{array}$} & \multicolumn{2}{|c|}{$\begin{array}{l}\text { Not achieved FBG } \\
\text { target (B) } \mathrm{N}=27\end{array}$} & \multirow[b]{2}{*}{$\mathrm{p}$} \\
\hline & $\%$ & $\mathrm{Sd}$ & $\%$ & sd & \\
\hline Breakfast & $20 \cdot 4$ & $7 \cdot 3$ & $21 \cdot 6$ & $11 \cdot 7$ & $0 \cdot 9$ \\
\hline Morning snack \& drinks & $7 \cdot 6$ & $7 \cdot 9$ & $8 \cdot 7$ & $12 \cdot 4$ & $0 \cdot 7$ \\
\hline Lunch & $27 \cdot 1$ & $9 \cdot 0$ & $26 \cdot 2$ & $11 \cdot 9$ & 0.7 \\
\hline Afternoon snack \& drinks & $8 \cdot 0$ & $6 \cdot 6$ & $6 \cdot 9$ & $6 \cdot 3$ & $0 \cdot 3$ \\
\hline Dinner & $30 \cdot 3$ & $11 \cdot 0$ & $33 \cdot 5$ & $12 \cdot 2$ & $0 \cdot 1$ \\
\hline Evening snack \& drinks & $6 \cdot 5$ & $7 \cdot 5$ & $3 \cdot 3$ & $5 \cdot 5$ & $0 \cdot 01$ \\
\hline
\end{tabular}

Those who achieved FBG targets (A) were more likely to consume three meals a-day and snack in each of the three snacking periods than those who did not (B) (3 meals a day: $92 \% \mathrm{v} .78 \%, p=0.04 ; 3$ snacking period: $11 \%$ v. $4 \%, p=0.05)$. In terms of distribution of $\mathrm{CHO}$ and energy across meals and snacks, significant differences between groups A \& B were only found for evening snacks and drinks: women achieving FBG targets (group A) consumed a higher percentage of their carbohydrates $(p=0 \cdot 01)$ and energy $(p=$ 0.03 ) from evening snacks and drinks (see table). Furthermore, group A were more likely to consume $>20$ g of carbohydrate in evening snacks $(\mathrm{p}=0.02)$. No other significant differences were found in relation to the good practise advice. No significant differences were found comparing women achieving post-breakfast blood glucose targets and those who did not.

In conclusion, increased evening snacking was associated with achieving fasting blood glucose targets in this pilot study; however this was contrary to clinical expectations.

myfood24 was developed with an MRC Grant G1100235.

1. Petry CJ (2010) Br J Nutr 104, 775-87.

2. NICE (2015) https://www.nice.org.uk/guidance/ng3/

3. Uplinger N (2009) Curr Diab Rep 4, 291-5.

4. Carter MC, Albar SA, Morris MA et al. (2015) Nutrients 7, 4016-4032. 